\title{
Effect of Acidity on Optical Properties of Isolated Skeletal Muscle Fibers
}

\section{H.J. Swatland}

\author{
University of Guelph, Dept. Food Science, Guelph, Ontario, N1G 2W1
}

Extremely pale meat is unattractive, looses fluid and is difficult to use for secondary processing. The problem is worst in pork, but also occurs in poultry. If anaerobic glycolysis is rapid or extended, lowangle x-ray diffraction shows fluid is released from the myofilament lattice, where lateral electrostatic repulsion is minimal at the isoelectric point of muscle proteins [1]. But what causes paleness at a low $\mathrm{pH}$ ? There are two common explanations. Paleness originates from scattering by precipitated sarcoplasmic proteins [2], and shrinkage of the myofilament lattice at a low $\mathrm{pH}$ increases reflection at the myofibrillar surface [3]. A third possibility considered here is that muscle fiber refraction contributes to paleness. If refraction is weak, the original directionality of the light may be maintained so light passes deep into the meat rather than being scattered back to the surface to appear as paleness. If refraction is strong, directionality may be lost, refractive scattering may be increased, so meat appears pale. To test this working hypothesis, experiments were undertaken on individual muscle fibers in a microscope chamber perfused with $0.2 \mathrm{M}$ phosphate buffer.

After washing out myoglobin, transmittance $(T)$ spectra through 20 pork fibers were measured from 300 to $800 \mathrm{~nm}$ at high and low $\mathrm{pH}(\mathrm{pH} 7.26 \pm 0.15$ and $5.49 \pm 0.06)$, starting half the samples at low and half at high $\mathrm{pH}$. As fibers were moved across the measuring aperture, light at short wavelengths was first to be refracted through the fiber and lost by scattering. Finally, with all wavelengths traversing the full depth of the fiber, $T$ was higher at high than low $\mathrm{pH}(300$ to $740 \mathrm{~nm}, \mathrm{P}<0.05 ; 310$ to $510 \mathrm{~nm}, \mathrm{P}<0.01)$. The Becke line was detected in many fibers as a bright line caused by refraction at the edge of the fiber [4].

One would not necessarily expect the photometric laws of Lambert, Beer and Bouguer to hold true for microscopic distances through strongly scattering fibers, so pork fibers $(\mathrm{n}=50)$ were examined at $\mathrm{pH}$ 5.5 or 7.0 with a mechanical scanning stage and photometer. The objective was to measure $T$ in relation to fiber diameter, anticipating attenuation of $T$ with increasing diameter. However, $T$ across fibers was often asymmetrical, showing fibers retained flattened sides after being released from bulk pork. In bulk pork, the range in flattened sides is from 3 to 8, with a mode of 5 or 6 sides, depending on sample origin within a muscle [5]. A lateral measurement of fiber diameter $(89.76 \pm 17.07 \mu \mathrm{m}$ at $\mathrm{pH} 5.5$ and $97.43 \pm$ $20.04 \mu \mathrm{m}$ at $\mathrm{pH} 7.0, \mathrm{P}>0.05)$ was compared with minimum $T$ at any point across a fiber. No $T$ attenuation was detected at $\mathrm{pH} 7.0(\mathrm{r}=0.07)$. At $\mathrm{pH} 5.5$, instead of attenuation, the relationship of $T$ with diameter was unexpectedly positive $(\mathrm{r}=0.44, \mathrm{P}<0.05)$, possibly from differences in myofibrillar composition between small and large diameter fibers (fibrillenstruktur versus felderstruktur). However, attenuation at $\mathrm{pH} 5.5$ was greater than at $\mathrm{pH} 7.0\left(2.76 \pm 1.19\right.$ vs $1.42 \pm 0.65 \Delta T \mathrm{~mm}^{-1}$, respectively, $\mathrm{P}<$ $0.001)$ and was strongly related to wavelength $(\mathrm{r}=0.97, \mathrm{P}<0.001)$. Thus, fiber shape and physiological fiber-type composition may affect optical properties of bulk meat. Results agreed with myofibrillar refraction contributing to 
post-mortem development of normal paleness in pork.

To check these findings were applicable to bulk meat, bulk refractive index $(n)$ was measured with an Abbe refractometer using a red laser for $T$ and a green laser for reflectance. The critical angle, although obscured by scattering, was detected subjectively at the red-green boundary. The refractometer also was operated under computer control, detecting $n$ photometrically. Pork longissimus thoracis $(\mathrm{n}=20)$ had higher $n$ than biceps femoris $(1.357 \pm 0.004$ versus $1.352 \pm 0.005$, respectively, $\mathrm{P}<0.001)$. Japanese pork colour scores $(J P C S)$ are widely used in the meat industry and range from 1 (very pale) to 5 (very dark). Longissimus thoracis had lower JPCS than biceps femoris $(2.92 \pm 0.37$ versus $3.87 \pm 0.48$, respectively, $\mathrm{P}<0.001)$. In pooled samples $(\mathrm{n}=40), n$ was correlated with $J P C S, \mathrm{r}=-0.55, \mathrm{P}<0.001$. Thus, the negative slope showed pale pork had a higher refractive index than dark pork, as anticipated from microscopy of single fibers.

Deviations from the photometric laws in bulk meat were examined using optical fibers mounted in gauge- 13 hypodermic needles with a $15^{\circ}$ tip. The tip angle modified the cone of illumination produced by the optical fiber, but the elliptical window still responded in a logical manner to test objects. $T$ spectra obtained as needles moved apart to lengthen the light path through meat were atypical, being almost flat. Pushing needles into the meat to shorten the light path, however, yielded typical spectra for meat, with a secondary absorbance band at $550 \mathrm{~nm}$ and low absorbance at $700 \mathrm{~nm}$. The effect of optical path length through the meat was detectable $(\mathrm{P}<0.01)$ for all visible wavelengths, but the magnitude of change was greater at $700 \mathrm{~nm}$ than at $400 \mathrm{~nm}$. $T$ measured perpendicularly through pork muscle fibers was a linear, arithmetic function of path length (slope $-0.103 \pm 0.038 \mathrm{~T} \mathrm{~cm}^{-1}$ at $700 \mathrm{~nm}, \mathrm{r}=-0.99, \mathrm{P}<0.001$ ) with no evidence of an exponential relationship predicted by the photometric laws.

Research in progress uses polarized light. In myofibrils, light splits into two components with different velocities, the ordinary ray $(O)$ and the extraordinary ray $(E)$, with $O \perp E$. Birefringence is given by $n_{E}-$ $n_{O}$. Retardation, the decrease in velocity of light caused by interaction with muscle proteins, is detected as phase retardation, the interference caused by path difference $E \neq O$. The path difference of a depth of fiber $(\Gamma)$ is measured by ellipsometry with a de Sénarmont compensator [6], $\boldsymbol{\Gamma} \mathbf{n m}=\mathbf{K}_{\mathbf{\lambda}} \mathbf{n m}^{-\mathbf{1}^{\circ}}$. $\mathbf{u}^{\mathbf{o}}$ where $\mathrm{u}$ is the angle in degrees required for compensation, and $\mathrm{K}_{\lambda}$ is the monochromatic de Sénarmont constant or path difference for $1^{\circ}$ of rotation. Following the same logic as for measuring attenuation of $T$ in relation to fiber diameter, if the optical path length can be found as well as $\Gamma$, it may be possible to quantify the effect of acidity on birefringence, giving us an appreciation of the refractive contribution to meat paleness from the molecular to the bulk state. Preliminary results confirm that $\Gamma$ increases as $\mathrm{pH}$ decreases, but finding the exact relationship is difficult. Low molarity of the buffer makes it difficult to control fiber $\mathrm{pH}$, whereas equilibration to $0.2 \mathrm{M}$ buffer removes some actomyosin [7].

[1] H.J. Swatland et al., J. Anim. Sci. 67 (1989) 1465.

[2] J.R. Bendall and J. Wismer-Pedersen, J. Food Sci. 27 (1962) 144.

[3] R. Hamm, Adv. Food Res. 10 (1960) 355.

[4] R.C. Faust, Optical properties. In R. Meredith and J.W.S. Hearle, Physical Methods of Investigating Textiles (pp. 320-345). Textile Book Publishers, New York, 1959.

[5] H.J. Swatland, J. Anim. Sci. 41 (1975) 78.

[6] M. Pluta, M. Advanced Light Microscopy. Amsterdam: Elsevier. Vol. 1, p. 38. 1988.

[7] Research supported by a grant for basic research from the Danish Bacon and Meat Council. 